\title{
TU/e EmonOWEN

\section{Deterministic all-optical switching of synthetic ferrimagnets using single femtosecond laser pulses}

\section{Citation for published version (APA):}

Lalieu, M. L. M., Peeters, M. J. G., Haenen, S. R. R., Lavrijsen, R., \& Koopmans, B. (2017). Deterministic alloptical switching of synthetic ferrimagnets using single femtosecond laser pulses. Physical Review B, 96(22), [220411(R)]. https://doi.org/10.1103/PhysRevB.96.220411

DOI:

10.1103/PhysRevB.96.220411

Document status and date:

Published: 26/12/2017

\section{Document Version:}

Publisher's PDF, also known as Version of Record (includes final page, issue and volume numbers)

\section{Please check the document version of this publication:}

- A submitted manuscript is the version of the article upon submission and before peer-review. There can be important differences between the submitted version and the official published version of record. People interested in the research are advised to contact the author for the final version of the publication, or visit the $\mathrm{DOI}$ to the publisher's website.

- The final author version and the galley proof are versions of the publication after peer review.

- The final published version features the final layout of the paper including the volume, issue and page numbers.

Link to publication

\section{General rights}

Copyright and moral rights for the publications made accessible in the public portal are retained by the authors and/or other copyright owners and it is a condition of accessing publications that users recognise and abide by the legal requirements associated with these rights.

- Users may download and print one copy of any publication from the public portal for the purpose of private study or research.

- You may not further distribute the material or use it for any profit-making activity or commercial gain

- You may freely distribute the URL identifying the publication in the public portal.

If the publication is distributed under the terms of Article 25fa of the Dutch Copyright Act, indicated by the "Taverne" license above, please follow below link for the End User Agreement:

www.tue.nl/taverne

Take down policy

If you believe that this document breaches copyright please contact us at:

openaccess@tue.nl

providing details and we will investigate your claim. 


\title{
Deterministic all-optical switching of synthetic ferrimagnets using single femtosecond laser pulses
}

\author{
M. L. M. Lalieu, ${ }^{*}$ M. J. G. Peeters, S. R. R. Haenen, R. Lavrijsen, and B. Koopmans \\ Department of Applied Physics, Institute for Photonic Integration, Eindhoven University of Technology, \\ P.O. Box 513, 5600 MB Eindhoven, The Netherlands
}

(Received 12 November 2017; published 26 December 2017)

\begin{abstract}
We experimentally demonstrate single-pulse all-optical switching in $\mathrm{Pt} / \mathrm{Co} / \mathrm{Gd}$ stacks using linearly polarized laser pulses. This shows that thermal single-pulse switching is not limited to ferrimagnetic alloys, but is also possible in ferrimagnetic multilayers that are highly suitable for future applications due to easy fabrication and (interface) engineering. Moreover, it is shown that the threshold fluence needed for the optical switch strongly depends on the thickness of the Co layer, with a remarkable low threshold fluence of $\approx 1.2 \mathrm{~mJ} / \mathrm{cm}^{2}$ for a Co thickness of $0.8 \mathrm{~nm}$. Lastly, helicity-dependent measurements showed no significant effect of the magnetic circular dichroism in these thin magnetic layers.
\end{abstract}

DOI: 10.1103/PhysRevB.96.220411

All-optical switching (AOS) of the magnetization was first observed in ferrimagnetic GdFeCo alloys. At first, the switch was found to be helicity dependent [1], which was explained by means of an effective magnetic field created via the inverse Faraday effect. A few years later, it was shown that for this material the helicity dependence results from magnetic circular dichroism [2], and purely thermal toggle switching using linearly polarized laser pulses was demonstrated [3]. Insight into this thermal switching mechanism was obtained using time-resolved element-specific x-ray magnetic circular dichroism [4]. These measurements revealed that the ultrafast switch is governed by the large difference in demagnetization rates in combination with the antiferromagnetic (AF) exchange interaction between the rare-earth (RE) and transition-metal (TM) magnetic sublattices [4-7]. The thermal single-pulse switching mechanism has only been observed in RE-TM alloys, but is also predicted for synthetic ferrimagnetic multilayers [8,9]. A second mechanism, helicity-dependent multiple-pulse switching, has been found in a wider variety of magnetic materials [10-13], typically needing tens of pulses for the switch [12].

All-optical switching has gained a lot of attention due to its high potential for fast and energy-efficient writing in future data storage devices. An appealing concept would be to combine AOS with the racetrack memory [14], as illustrated in Fig. 1(a). To fully benefit from the speed and energy efficiency of AOS, single-pulse switching is necessary. At the moment, however, the thermal single-pulse AOS is only observed in RE-TM alloys, requiring a relatively complex fabrication process. In this Rapid Communication, we experimentally demonstrate helicity-independent single-pulse toggle switching in $\mathrm{Pt} / \mathrm{Co} / \mathrm{Gd}$ stacks. This structure is chosen because of the AF coupling [15] and the large contrast in demagnetization times [16,17] for the $\mathrm{Co}$ and $\mathrm{Gd}$ in the $\mathrm{Co} / \mathrm{Gd}$ bilayer, and the presence of a perpendicular magnetic anisotropy due to the Pt seed layer. Our observations show that thermal single-pulse switching is not limited to the RE-TM

\footnotetext{
*Corresponding author: m.1.m.lalieu@tue.nl
}

alloys, but is also possible in easy to fabricate and engineer synthetic ferrimagnetic multilayers. Also, we show that the minimum energy needed for AOS in these $\mathrm{Pt} / \mathrm{Co} / \mathrm{Gd}$ stacks can be controlled by tuning the Co layer thickness. It is noted that high domain wall velocities have been demonstrated in similar $\mathrm{Pt} / \mathrm{Co} / \mathrm{Gd}$ stacks, attributed to the Dzyaloshinskii-Moriya interaction [15], which further substantiates the applicability of AOS in future memory devices.

The measurements are performed on $\mathrm{Si}: \mathrm{B}$ (substrate)/Ta(4)/ $\mathrm{Pt}(4) / \mathrm{Co}\left(t_{\mathrm{Co}}\right) / \mathrm{Gd}(3) / \mathrm{Pt}(2)$ stacks (thickness in nanometers), which are deposited using dc magnetron sputtering $\left(10^{-7}-10^{-8}\right.$ mbar base pressure) at room temperature. In this Rapid Communication, we focus on a series of samples with $t_{\mathrm{Co}}=0.8,1.0,1.2$, and $1.4 \mathrm{~nm}$. All Co thicknesses result in a perpendicular magnetic anisotropy (PMA). A polar magneto-optical Kerr effect measurement on the $t_{\mathrm{Co}}=1.0 \mathrm{~nm}$ stack is shown in the inset of Fig. 1(b) (see Supplemental Material Note I for all $t_{\mathrm{Co}}[18]$ ), showing a square hysteresis loop with $100 \%$ remanence, confirming a well-defined PMA.

To verify the $(\mathrm{AF})$ exchange interaction between the Co and Gd layers, the magnetic moment per unit area is measured as a function of temperature, extracted from hysteresis curves measured at different temperatures using a VSM-SQUID. Figure 1(b) shows the measurement performed on the $t_{\mathrm{Co}}=$ $1.0 \mathrm{~nm}$ stack. The AF coupling between the layers is demonstrated by the presence of a compensation temperature at $120 \mathrm{~K}$, at which the magnetic moments of the Co and Gd layers are equal and compensate each other due to an antiparallel alignment. Below (above) this temperature the magnetic moment of the $\mathrm{Gd}(\mathrm{Co})$ layer is larger.

The Curie temperature of bulk Gd lies just below room temperature. At the $\mathrm{Co} / \mathrm{Gd}$ interface, however, it is enhanced due to the exchange interaction with the Co. This results in an induced magnetization in the $\mathrm{Gd}$ near the interface at room temperature [see zoom-in Fig. 1(a)], as is seen in $\mathrm{Fe} / \mathrm{Gd}$ [19]. While some alloy formation at the interface is likely due to interdiffusion of $\mathrm{Co}$ and $\mathrm{Gd}$, an estimate of the amount of magnetic $\mathrm{Gd}$ at room temperature can be made assuming sharp interfaces, and using the data in Fig. 1(b). The analysis, presented in Supplemental Material Note II [18], 
(a)

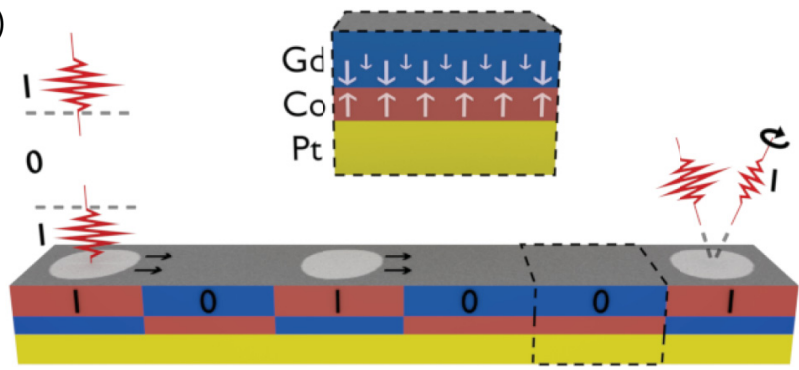

(b)

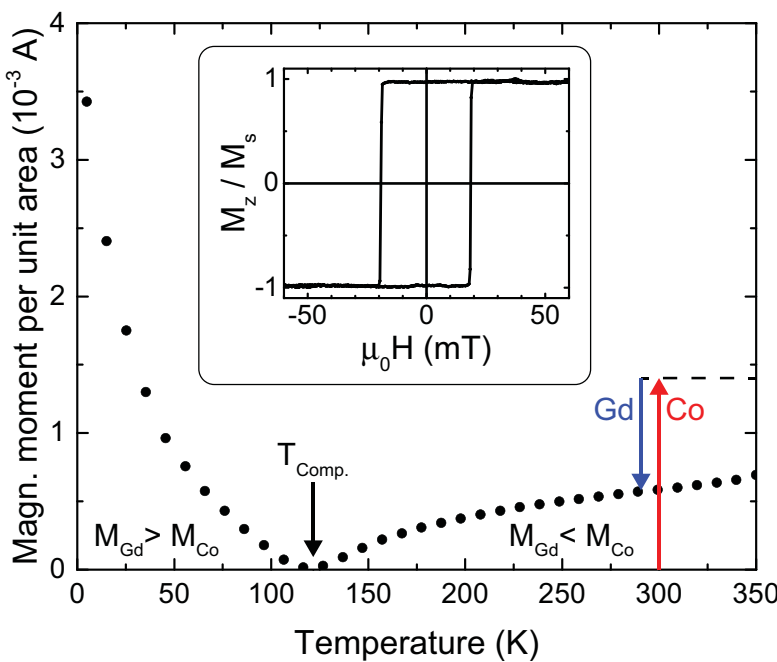

FIG. 1. (a) Illustration of the hybrid memory device, in which magnetic data is written into a $(\mathrm{Pt} / \mathrm{Co} / \mathrm{Gd})$ racetrack using AOS (left), and readout all-optically using the magneto-optical Kerr effect (right). Exploiting the PMA in the stack in combination with the Dzyaloshinskii-Moriya interaction [15], data can be transported efficiently through the racetrack using the spin Hall effect originating in a $(\mathrm{Pt})$ heavy metal seed layer [25]. Grayscale topside of the racetrack displays Kerr images from an actual AOS measurement performed on the $\mathrm{Pt} / \mathrm{Co} / \mathrm{Gd}$ stack. Zoom-in shows the $\mathrm{AF}$ coupled Co and Gd layers, and the local magnetization near the interface in the Gd at room temperature. (b) VSM-SQUID measurement of the magnetic moment per unit area as a function of the temperature for the $\mathrm{Pt} / \mathrm{Co} / \mathrm{Gd}$ stack with $t_{\mathrm{Co}}=1.0 \mathrm{~nm}$. Compensation temperature at $120 \mathrm{~K}$ demonstrates the AF exchange interaction between the $\mathrm{Co}$ and Gd layers. Inset: Polar magneto-optical Kerr effect measurement performed at room temperature on the same structure. The square hysteresis loop with $100 \%$ remanence confirms a well-defined PMA.

shows a magnetic moment corresponding to $0.45 \mathrm{~nm}$ of fully saturated $\mathrm{Gd}$ at $300 \mathrm{~K}$, which is similar as found in previous work [15].

After having confirmed the synthetic ferrimagnetic behavior, we examine the dynamic response of the magnetization in the $\mathrm{Pt} / \mathrm{Co} / \mathrm{Gd}$ stacks on laser-pulse excitation. In these measurements, performed at room temperature, the magnetization is first saturated with an external field, whereafter the field is turned off and the structure is exposed to linearly polarized laser pulses with a central wavelength of $700 \mathrm{~nm}$ and a pulse duration of $\approx 100 \mathrm{fs}$. The response of the magnetization on the laser-pulse excitation is measured in the steady state (i.e., long after the excitation) using a dedicated magneto-optical Kerr microscope.

Figure 2(a) shows the result of the measurement performed on the $t_{\mathrm{Co}}=1.0 \mathrm{~nm}$ stack, in which ten separate spots are excited by a different number of subsequent laser pulses. The figure shows the results for both up (top) and down (bottom) initial magnetization directions, where the dark and light regions correspond to the magnetization pointing up and down, respectively. The labels indicate the number of pulses each spot is exposed to. For the spots that are excited by a single laser pulse, we observe that for both initial magnetization directions a homogeneous domain with the magnetization in the opposite direction is written [see also Fig. 2(b) for zoom-in]. This demonstrates that we have single-pulse helicity-independent AOS in the $\mathrm{Pt} / \mathrm{Co} / \mathrm{Gd}$ stack.

As a reference measurement, the same single-pulse measurement has been performed on a $\mathrm{Si}: \mathrm{B} / \mathrm{Ta}(4) / \mathrm{Pt}(4) / \mathrm{Co}(1) /$ $\mathrm{Pt}(2)$ structure, i.e., without the Gd layer. The result is shown in Fig. 2(c). In this case, a multidomain state is created after the excitation, as found in previous work [12]. A pulse-energy-dependent measurement shows the creation of a multidomain state down to the threshold pulse energy (see Supplemental Material Note III [18]). The absence of single-pulse $\mathrm{AOS}$ in the $\mathrm{Pt} / \mathrm{Co} / \mathrm{Pt}$ stack demonstrates the necessity of the $\mathrm{Co} / \mathrm{Gd}$ combination for the switch.

Next, we explore the effect of consecutive laser pulses on the magnetization by examining the spots in Fig. 2(a) where the structure is exposed to multiple pulses. For two pulses, no net magnetization reversal is observed, showing that the complete domain written by the first pulse is switched back by the second pulse. Analogously, every odd number of pulses leaves a switched domain, whereas an even number of pulses results in no net reversal. Although the figure already shows a complete toggle up to ten pulses, additional measurements have shown deterministic toggle switching for over 5000 pulses. The observed switching behavior is consistent with the thermal single-pulse switching mechanism discussed earlier.

In Fig. 2(e) we investigate the effect of a domain wall being present in the switching area by exciting the structure with three partly overlapping laser pulses. The figure shows a switched domain in the areas where there is no overlap between the pulses ("1"), and where all three pulses overlap ("3"). At the regions where only two pulses overlap ("2") no net switch is observed, again demonstrating a toggle behavior. Moreover, it shows that the domain wall stays intact, and the domains at either side toggle in opposite directions.

For AOS to be used in future memory devices it is important to minimize the energy per unit area needed to switch the magnetization, i.e., the threshold fluence. In the case of the thermal switching mechanism, the threshold fluence depends on the energy needed to fully demagnetize the TM layer, which in turn depends on its Curie temperature. Considering the decrease in Curie temperature with film thickness for Co films in the thin-film limit, a reduction in the threshold fluence can be expected for decreasing $t_{\mathrm{Co}}$ in the $\mathrm{Pt} / \mathrm{Co} / \mathrm{Gd}$ stack. Therefore, the Co thickness dependence of the threshold 

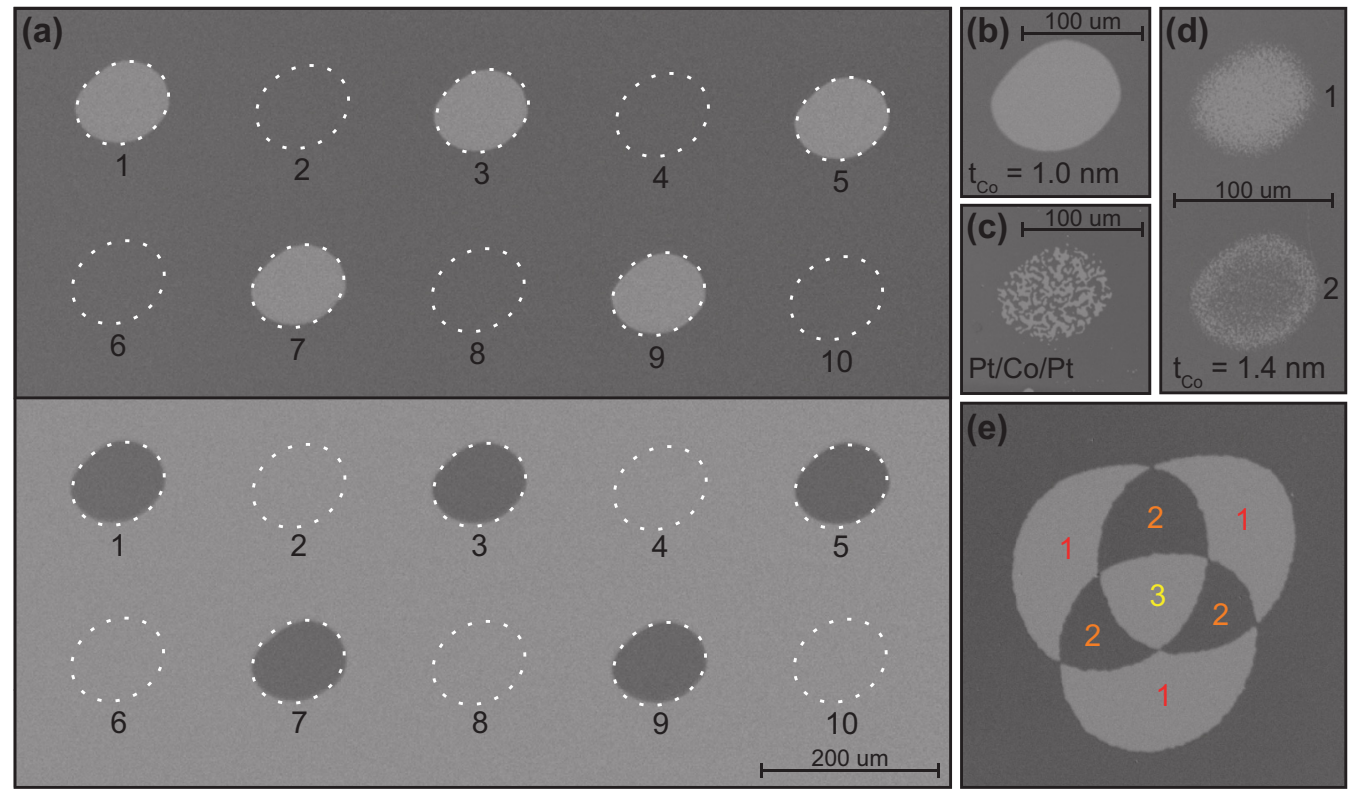

FIG. 2. AOS measurements performed on Pt/Co/Gd stacks. (a) Switching experiments performed on the $t_{\mathrm{Co}}=1.0 \mathrm{~nm}$ stack for both initial saturation directions. The numbered regions (dotted circles) correspond to the spots where the structure is excited by the laser (fluence $4.2 \mathrm{~mJ} / \mathrm{cm}^{2}$ ), where the labels indicate the number of pulses each spot is exposed to. (b)-(d) Zoom-in on single-pulse measurements performed on the $t_{\mathrm{Co}}=1.0 \mathrm{~nm}$ stack (b) and a Pt/Co/Pt stack (c). (d) Single (top) and two-pulse (bottom) measurements performed on the $t_{\mathrm{Co}}=1.4 \mathrm{~nm}$ stack (fluence $5.6 \mathrm{~mJ} / \mathrm{cm}^{2}$ ). (e) AOS measurement with three partly overlapping pulses. The numbers in the different regions correspond to the number of pulses the region has been exposed to.

fluence is investigated, which is done by measuring the size of the written domain as a function of pulse energy. In this measurement the sample is excited by single laser pulses with different pulse energies. Figure 3(a) shows the measurement performed on the $t_{\text {Co }}=1.0 \mathrm{~nm}$ stack. The figure shows an onset of AOS at a pulse energy of $280 \mathrm{~nJ}$. Above this value, the domain size increases with pulse energy, as is expected for a spatial Gaussian-shaped pulse. Measurements at very high fluences show the formation of a multidomain state in the center area of the domain (not shown). At these fluences the lattice temperature at the center of the Gaussian pulse rises above the Curie temperature, after which a multidomain state is formed during the successive slow cooldown [1].

The threshold fluence is defined as the threshold pulse energy divided by the laser-spot area (using the FWHM of a Gaussian distribution as the spot diameter). Both the threshold pulse energy and laser-spot area are determined using the size of the switched domain as a function of the pulse energy, shown in Fig. 3(b) for $t_{\mathrm{Co}}=0.8,1.0$, and $1.2 \mathrm{~nm}$ (solid symbols). Following the Liu method [20] (and taking into account the slight elliptical shape of the laser spot), the relation between the domain size and the pulse energy $P$ can be derived to be

$$
\text { Domain size }=2 \pi r \sigma^{2} \ln \left(\frac{P}{P_{0}}\right),
$$

in which $P_{0}$ is the threshold pulse energy, $\sigma$ the standard deviation along the short axis of the slightly elliptical Gaussian pulse, and $r$ the (constant) ratio between the $\sigma$ 's along the long and short axis $\left(\sigma_{\text {long }}=r \sigma\right)$. With $r$ determined from the Kerr images, both $\sigma$ and $P_{0}$ can be obtained by fitting the data using Eq. (1). From these values the threshold fluence $F_{0}$ is calculated, and shown in the inset of Fig. 3(b). A strong Co thickness dependence of the threshold fluence is observed, decreasing for smaller $t_{\mathrm{Co}}$. This indicates that the full magnetic structure participates in the AOS. At the lowest thickness, $t_{\mathrm{Co}}=0.8$, a threshold fluence of $1.2 \mathrm{~mJ} / \mathrm{cm}^{2}$ is found, which is significantly lower than the threshold fluence found for singlepulse AOS in GdFeCo alloys [2].

The open symbols in Fig. 3(b) correspond to the $t_{\mathrm{Co}}=$ $1.4 \mathrm{~nm}$ stack. Although the threshold fluence is in line with those found for the thinner Co layers, the actual written domains are not. Figure 2(d) (top) shows that for this structure a speckled domain is written, instead of a homogeneous domain found for the other stacks. The pulse-energy-dependent measurement shows this behavior for all pulse energies (see Supplemental Material Note IV [18]). The speckled domain is believed to be the result of the structure being near the spin reorientation limit, observed by the change from a domain wall propagation dominated reversal for small $t_{\mathrm{Co}}$ to a nucleation dominated reversal for the $t_{\mathrm{Co}}=1.4 \mathrm{~nm}$ structure during field sweep measurements (see Supplemental Material Note I [18]). As a result of the more stochastic behavior, the magnetization only partly toggles back in a two-pulse experiment, as shown in Fig. 2(d) (bottom).

Lastly, helicity-dependent measurements were performed to investigate the effect of the magnetic circular dichroism (MCD). From previous measurements on GdFeCo alloys $[1,2]$, it is known that the polarization-dependent absorption 


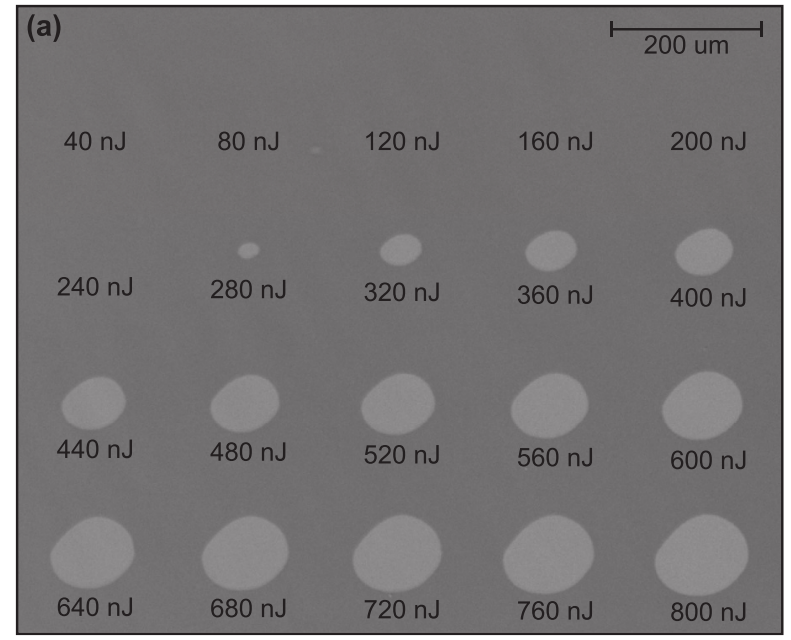

(b)

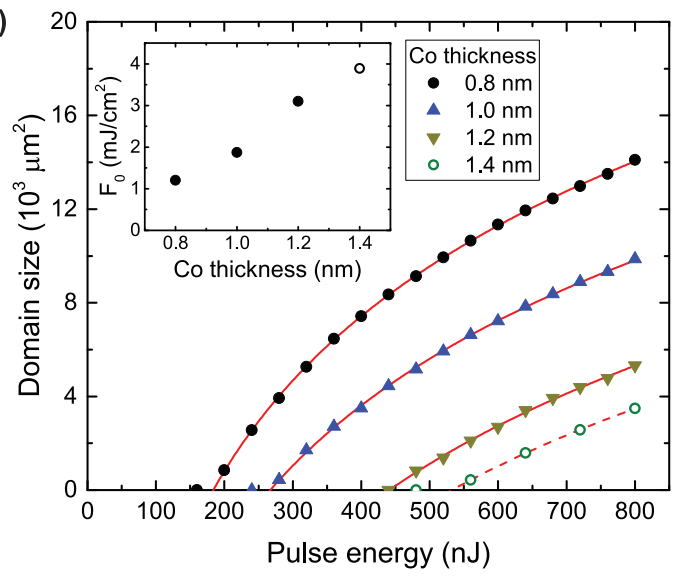

FIG. 3. Pulse-energy-dependent AOS measurements performed on $\mathrm{Pt} / \mathrm{Co} / \mathrm{Gd}$ stacks. (a) Kerr image of the measurement performed on the $t_{\mathrm{Co}}=1.0 \mathrm{~nm}$ stack, showing an onset of AOS at $280 \mathrm{~nJ}$ and an increase in domain size with pulse energy. Pulse energies are indicated in the figure. (b) Domain size as a function of pulse energy for stacks with $t_{\mathrm{Co}}=0.8,1.0,1.2$, and $1.4 \mathrm{~nm}$. Inset: Threshold fluence as a function of the Co thickness. opens up a fluence window where the AOS becomes helicity dependent, adding functionality for future applications. Measurements performed on the $t_{\mathrm{Co}}=1.0$ stack, however, show no significant effect of the MCD on the threshold pulse energy (see Supplemental Material Note V [18]). This could be the result of the limited thickness of the magnetic layer, in which the MCD is generated, accounting for only $10 \%$ of the full stack participating in the absorption of the laser pulse.

Examining the AOS behavior observed in the $\mathrm{Pt} / \mathrm{Co} / \mathrm{Gd}$ stacks, it is consistent with the thermal single-pulse switching found in RE-TM alloys. Also, the Co:Gd (magnetic) thickness ratio found for the $\mathrm{Pt} / \mathrm{Co} / \mathrm{Gd}$ stack is 0.69:0.31 [Fig. 1(b)], which is within the range for which thermal AOS has been predicted in Fe/Gd bilayers [9], where the local exchange interaction at the interface is used to drive the switch. However, due to the localized nature of the magnetic layers in the $\mathrm{Pt} / \mathrm{Co} / \mathrm{Gd}$ stack, nonlocal transfer of angular momentum is expected to occur as well [21-23], possibly contributing or even initiating the switch [24]. Using additional interface engineering in the ferrimagnetic multilayers used here, e.g., adding dusting or spacer layers, more insight into the importance of the different mechanisms can be obtained in the future.

In summary, we have experimentally demonstrated singlepulse helicity-independent toggle switching in $\mathrm{Pt} / \mathrm{Co} / \mathrm{Gd}$ stacks. The observation of single-pulse AOS in ferrimagnetic multilayers with built-in DMI and suitable for spin Hall effect driven domain wall motion provides an important step toward the integration of AOS in future memory devices. Moreover, it was found that the threshold fluence scales with the thickness of the Co layer, going as low as $1.2 \mathrm{~mJ} / \mathrm{cm}^{2}$, which scales down to below $50 \mathrm{fJ}$ needed to switch a $50 \times 50 \mathrm{~nm}^{2}$ bit.

This work is part of the Gravitation program "Research Centre for Integrated Nanophotonics," and the research program of the Foundation for Fundamental Research on Matter (FOM), which are financed by the Netherlands Organisation for Scientific Research (NWO).
[1] C. D. Stanciu, F. Hansteen, A. V. Kimel, A. Kirilyuk, A. Tsukamoto, A. Itoh, and Th. Rasing, All-Optical Magnetic Recording with Circularly Polarized Light, Phys. Rev. Lett. 99, 047601 (2007).

[2] A. R. Khorsand, M. Savoini, A. Kirilyuk, A. V. Kimel, A. Tsukamoto, A. Itoh, and Th. Rasing, Role of Magnetic Circular Dichroism in All-Optical Magnetic Recording, Phys. Rev. Lett. 108, 127205 (2012).

[3] T. A. Ostler, J. Barker, R. F. L. Evans, R. W. Chantrell, U. Atxitia, O. Chubykalo-Fesenko, S. El Moussaoui, L. Le. Guyader, E. Mengotti, L. J. Heyderman, F. Nolting, A. Tsukamoto, A. Itoh, D. Afanasiev, B. A. Ivanov, A. M. Kalashnikova, K. Vahaplar, J. Mentink, A. Kirilyuk, Th. Rasing, and A. V. Kimel, Ultrafast heating as a sufficient stimulus for magnetization reversal in a ferrimagnet, Nat. Commun. 3, 666 (2012).

[4] I. Radu, K. Vahaplar, C. Stamm, T. Kachel, N. Pontius, H. A. Durr, T. A. Ostler, J. Barker, R. F. L. Evans, R. W. Chantrell, A. Tsukamoto, A. Itoh, A. Kirilyuk, Th. Rasing, and A. V. Kimel,
Transient ferromagnetic-like state mediating ultrafast reversal of antiferromagnetically coupled spins, Nature (London) 472, 205 (2011).

[5] J. H. Mentink, J. Hellsvik, D. V. Afanasiev, B. A. Ivanov, A. Kirilyuk, A. V. Kimel, O. Eriksson, M. I. Katsnelson, and Th. Rasing, Ultrafast Spin Dynamics in Multisublattice Magnets, Phys. Rev. Lett. 108, 057202 (2012).

[6] A. J. Schellekens and B. Koopmans, Microscopic model for ultrafast magnetization dynamics of multisublattice magnets, Phys. Rev. B 87, 020407 (2013).

[7] S. Wienholdt, D. Hinzke, K. Carva, P. M. Oppeneer, and U. Nowak, Orbital-resolved spin model for thermal magnetization switching in rare-earth-based ferrimagnets, Phys. Rev. B 88, 020406 (2013).

[8] R. F. L. Evans, T. A. Ostler, R. W. Chantrell, I. Radu, and Th. Rasing, Ultrafast thermally induced magnetic switching in synthetic ferrimagnets, Appl. Phys. Lett. 104, 082410 (2014). 
[9] S. Gerlach, L. Oroszlany, D. Hinzke, S. Sievering, S. Wienholdt, L. Szunyogh, and U. Nowak, Modeling ultrafast all-optical switching in synthetic ferrimagnets, Phys. Rev. B 95, 224435 (2017).

[10] S. Mangin, M. Gottwald, C.-H. Lambert, D. Steil, V. Uhlr, L. Pang, M. Hehn, S. Alebrand, M. Cinchetti, G. Malinowski, Y. Fainman, M. Aeschlimann, and E. E. Fullerton, Engineered materials for all-optical helicity-dependent magnetic switching, Nat. Mater. 13, 286 (2014).

[11] C-H. Lambert, S. Mangin, B. S. D. Ch. S. Varaprasad, Y. K. Takahashi, M. Hehn, M. Cinchetti, G. Malinowski, K. Hono, Y. Fainman, M. Aeschlimann, and E. E. Fullerton, All-optical control of ferromagnetic thin films and nanostructures, Science 345, 1337 (2014).

[12] M. S. El. Hadri, P. Pirro, C.-H. Lambert, S. Petit-Watelot, Y. Quessab, M. Hehn, F. Montaigne, G. Malinowski, and S. Mangin, Two types of all-optical magnetization switching mechanisms using femtosecond laser pulses, Phys. Rev. B 94, 064412 (2016).

[13] M. S. El Hadri, M. Hehn, P. Pirro, C.-H. Lambert, G. Malinowski, E. E. Fullerton, and S. Mangin, Domain size criterion for the observation of all-optical helicity-dependent switching in magnetic thin films, Phys. Rev. B 94, 064419 (2016).

[14] S. S. P. Parkin, M. Hayashi, and L. Thomas, Magnetic domainwall racetrack memory, Science 320, 190 (2008).

[15] T. H. Pham, J. Vogel, J. Sampaio, M. Vanatka, J.-C. RojasSanchez, M. Bonfim, D. S. Chaves, F. Choueikani, P. Ohresser, E. Otero, A. Thiaville, and S. Pizzini, Very large domain wall velocities in $\mathrm{Pt} / \mathrm{Co} / \mathrm{GdOx}$ and $\mathrm{Pt} / \mathrm{Co} / \mathrm{Gd}$ trilayers with Dzyaloshinskii-Moriya interaction, Europhys. Lett. 113, 67001 (2016).

[16] B. Koopmans, G. Malinowski, F. D. Longa, D. Steiauf, M. Fahnle, T. Roth, M. Cinchetti, and M. Aeschlimann, Explaining the paradoxical diversity of ultrafast laser-induced demagnetization, Nat. Mater. 9, 259 (2010).

[17] M. Wietstruk, A. Melnikov, C. Stamm, T. Kachel, N. Pontius, M. Sultan, C. Gahl, M. Weinelt, H. A. Dürr, and U. Bovensiepen, Hot-Electron-Driven Enhancement of Spin-Lattice Coupling in Gd and $\mathrm{Tb}$ 4f Ferromagnets Observed by Femtosecond X-Ray
Magnetic Circular Dichroism, Phys. Rev. Lett. 106, 127401 (2011).

[18] See Supplemental Material at http://link.aps.org/supplemental/ 10.1103/PhysRevB.96.220411 for (I) polar magneto-optical Kerr effect measurements on the $\mathrm{Pt} / \mathrm{Co} / \mathrm{Gd}$ stacks for all $\mathrm{Co}$ thicknesses; (II) analysis of the SQUID-VSM measurement on a Pt/Co/Gd stack; (III) pulse-energy-dependent AOS measurement on a $\mathrm{Pt} / \mathrm{Co} / \mathrm{Pt}$ stack; (IV) pulse-energy-dependent AOS measurement on a $\mathrm{Pt} / \mathrm{Co} / \mathrm{Gd}$ stack with $1.4 \mathrm{~nm}$ Co layer; and (V) helicity-dependent measurement on a $\mathrm{Pt} / \mathrm{Co} / \mathrm{Gd}$ stack.

[19] D. Haskel, G. Srajer, J. C. Lang, J. Pollmann, C. S. Nelson, J. S. Jiang, and S. D. Bader, Enhanced Interfacial Magnetic Coupling of Gd/Fe Multilayers, Phys. Rev. Lett. 87, 207201 (2001).

[20] J. M. Liu, Simple technique for measurements of pulsed Gaussian-beam spot sizes, Opt. Lett. 7, 196 (1982).

[21] G. Malinowski, F. D. Longa, J. H. H. Rietjens, P. V. Paluskar, R. Huijink, H. J. M. Swagten, and B. Koopmans, Control of speed and efficiency of ultrafast demagnetization by direct transfer of spin angular momentum, Nat. Phys. 4, 855 (2008).

[22] M. Battiato, K. Carva, and P. M. Oppeneer, Superdiffusive Spin Transport as a Mechanism of Ultrafast Demagnetization, Phys. Rev. Lett. 105, 027203 (2010).

[23] A. Melnikov, I. Razdolski, T. O. Wehling, E. Th. Papaioannou, V. Roddatis, P. Fumagalli, O. Aktsipetrov, A. I. Lichtenstein, and U. Bovensiepen, Ultrafast Transport of Laser-Excited SpinPolarized Carriers in $\mathrm{Au} / \mathrm{Fe} / \mathrm{MgO}(001)$, Phys. Rev. Lett. 107, 076601 (2011).

[24] C. E. Graves, A. H. Reid, T. Wang, B. Wu, S. de Jong, K. Vahaplar, I. Radu, D. P. Bernstein, M. Messerschmidt, L. Müller, R. Coffee, M. Bionta, S. W. Epp, R. Hartmann, N. Kimmel, G. Hauser, A. Hartmann, P. Holl, H. Gorke, J. H. Mentink, A. Tsukamoto, A. Fognini, J. J. Turner, W. F. Schlotter, D. Rolles, H. Soltau, L. Strüder, Y. Acremann, A. V. Kimel, A. Kirilyuk, Th. Rasing, J. Stöhr, A. O. Scherz, and H. A. Dürr, Nanoscale spin reversal by non-local angular momentum transfer following ultrafast laser excitation in ferrimagnetic GdFeCo, Nat. Mater. 12, 293 (2013).

[25] K-S. Ryu, L. Thomas, S.-H. Yang, and S. Parkin, Chiral spin torque at magnetic domain walls, Nat. Nanotechnol. 8, 527 (2013). 